\title{
Death Wears a Dress
}

\author{
Tania De Rozario \\ University of British Columbia, Canada/Writer, Canada \& Singapore
}

\begin{abstract}
In many parts of Asia, female ghosts play an interesting role in how the supernatural is imagined and constructed. Whether she be the pontianak who waits for her victim by the side of the road, or the mother or lover who returns for revenge, the female ghost is often characterised as treading the line between agency and oppression. On one hand, she is an autonomous character who seeks justice on her own terms; on another, she is usually reduced to a victim of violence while she is alive, and her agency is only granted in death... in the transformation of her identity from victim to villain.

Death Wears a Dress is a collection of poems inspired by numerous female "monsters" central to Asian folklore, many of whom continue to reincarnate through horror films, pop culture and social media. Through poetic verse, I hope to centralise, re-imagine and humanise the experiences, emotions, desires, fears and regrets of these fictitious women in an effort to unearth possible insights about gender, power, longing and justice.
\end{abstract}

Death Wears A Dress is being written with the support of Singapore's National Arts Council's Creation Grant.

Keywords: poetry, tropical, gothic, Asia, female ghosts 


\section{Appetite}

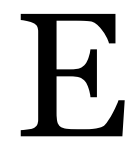

very object I see makes me hungry

all over again. Don't make me explain

this emptiness: just feed me

what you have, your moist meats

ripening with age, your root vegetables

dark with soil. I want to be buried

in your leisurely lunches, champagne

brunches, snacks snuck between

munches, suppers slurped

past midnight. Give me gravy

trains of staple grains, the cookie

that crumbles, the bigger fish

to fry. The big cheese, the bread

and butter, the bad eggs whose names

you mutter. Pass me the hot potato, hard

nut to crack, the souped-up smart cookies

selling themselves like hotcakes. I want

the bun in your oven, the apple of your eye,

one woman's meat and another's poison, candy

from a baby, then the baby itself, sweet-

toothed and teething on the milk

of human kindness. I want to make a meal

of you, your calves soft like veal, want

to butter you up then see if I feel

any fuller after eating your heart out

after making you my sweet

and just desserts. 


\section{On Losing One's Head}

$\mathrm{I}$ n a former life, l'd get angry. My husband would say Don't lose your head! In spite of indignation, some of that stuck, because now my head is all I am. Sure, other parts still linger neck down: heart, stomach, intestines, bits of skin... ....it is the rest of myself I lose these day. Every time the sun sets, I rise above my grave, look down at stray limbs, digits, genitals, wondering whether every woman is doomed in life to lose bits of themselves to someone else, scour endlessly the flesh of other people for something that makes them whole. 


\section{Motherhood}

7 his baby is the weight

of a neutron star: impossible

to cradle without breaking

oneself. Could you hold her

for a minute? Not so long, just until

her gravity becomes your coffin,

drags you down into the soil,

finds you buried alongside us.

The sky is heavy

with anxiety and rain.

No sacrifice is ever enough. 